\title{
Theoretical study of hydrogen bonding and proton transfer in the ground and lowest excited singlet states of tropolone
}

\author{
M. V. Vener and Steve Scheiner \\ Department of Chemistry, Southern Illinois University, Carbondale, Illinois 62901
}

\author{
N. D. Sokolov \\ Institute of Chemical Physics, RAS, 117334 Moscow, Russian Federation
}

(Received 13 June 1994; accepted 9 August 1994)

\begin{abstract}
Theoretical models of hydrogen bonding and proton transfer in the ground $\left(S_{0}\right)$ and lowest excited $\pi \pi^{*}$ singlet $\left(S_{1}\right)$ states of tropolone are developed in terms of the localized $\mathrm{OH} \cdots \mathrm{O}$ fragment model and $a b$ initio three-dimensional potential energy surfaces (PESs). The PESs for proton transfer in the $S_{0}$ and $S_{1}$ states are calculated using $a b$ initio SCF and CIS methods, respectively, with a 6-31G basis set which includes polarization functions on the atoms involved in the internal $H$ bond. The Schrödinger equation for nuclear vibrations is solved numerically using adiabatic separation of the variables. The calculated values for the $S_{0}$ state (geometry, relaxed barrier height, vibrational frequencies, tunnel splittings and H/D isotope effects) agree fairly well with available experimental and theoretical data. The calculated data for the $S_{1}$ state reproduce the principal experimental trends, established for $S_{1} \leftarrow S_{0}$ excitation in tropolone, but are less successful with other features of the dynamics of the excited state, e.g., the comparatively large value of vibrationless level tunnel splitting and its irregular increase with $\mathrm{O} \cdots \mathrm{O}$ excitation in $S_{1}$. In order to overcome these discrepancies, a model 2-D PES is constructed by fitting an analytical approximation of the CIS calculation to the experimental vibrationless level tunnel splitting and $\mathrm{O} \cdots \mathrm{O}$ stretch frequency of tropolone-OH. It is found that the specifics of the proton transfer in the $S_{1}$ state are determined by a relatively low barrier (only one doublet of the $\mathrm{OH}$ stretch lies under the barrier peak). Bending vibrations play a minor role in modulation of the proton transfer barrier, so correct description of tunnel splitting of the proton stretch levels in both electronic states can be obtained in terms of the two-dimensional stretching model, which includes $\mathrm{O} \cdots \mathrm{O}$ and $\mathrm{O}-\mathrm{H}$ stretching vibration coordinates only. 1994 American Institute of Physics.
\end{abstract}

\section{INTRODUCTION}

The intramolecular hydrogen bond in tropolone (TRN) has been studied extensively over the last two decades as it offers a model system in which to study proton transfer (PT) in the ground $\left(S_{0}\right)$ and first excited singlet $\pi \pi^{*}\left(S_{1}\right)$ states. $^{1}$ A consistent theoretical study of the vibrational dynamics of the hydrogen bond must take account of angular features, and requires consideration of a multidimensional potential energy surface (PES) for each electronic state. In contrast to malonaldehyde ${ }^{2,3}$ and the formic acid dimer, ${ }^{4}$ there is no previously published ab initio potential energy surface (PES) for TRN.

The present work considers the proton transfer in the $S_{0}$ and $S_{1}$ states of TRN by focusing explicitly on the $\mathrm{OH} \cdots \mathrm{O}$ fragment, ${ }^{5}$ via a three-dimensional $a b$ initio PES. The validity of this approximation for TRN is supported by experimental data. Sekiya et al. have recently ${ }^{6}$ shown that the $\nu_{14}^{\prime}$ low-frequency vibrational mode of TRN in its $S_{1}$ state couples strongly with $\mathrm{H}$ motion; more importantly, this mode is decoupled from the other degrees of freedom. The intramolecular normal coordinate $Q_{14}$ (symmetry $a^{\prime}$ ) corresponding to this vibration, "predominantly involves the displacements of the atoms in the $\mathrm{O}-\mathrm{H}-\mathrm{O}$ chelate ring" 6 and can be treated as a nearly pure $\mathrm{O} \cdots \mathrm{O}$ stretching vibration in terms of the localized $\mathrm{OH} \cdots \mathrm{O}$ fragment model. ${ }^{5}$

This paper first uses $a b$ initio methods to calculate the three-dimensional PES for proton transfer in the $S_{0}$ and $S_{1}$ states of TRN. Dynamics calculations next evaluate the effects of the vibrational excitation of the $0 \cdots \mathrm{O}$ stretching mode upon the transfer and lead to estimates of H/D isotope effects on the tunnel splitting of the two electronic states. The results are used to interpret the mechanism of enhancement of the tunneling probabilities by the low-frequency mode and clarify the specifics of the PT in the $S_{1}$ state.

\section{METHODS OF CALCULATION}

\section{General formulation}

There are two principal approaches to the theoretical study of proton transfer in $\mathrm{H}$-bonded systems containing the AHB fragment. The reaction surface Hamiltonian method ${ }^{2,3}$ treats the reaction pathway of PT in terms of simultaneous and complex movements of the entire molecular frame. Malonaldehyde, ${ }^{2,3}$ formic acid dimer, ${ }^{4}$ benzoic acid dimer, ${ }^{7}$ and other model systems ${ }^{8}$ have been examined in this manner. The second approach makes the approximation that the proton motion can be essentially decoupled from distant geometry changes, so that the reaction can be described in terms of the localized $\mathrm{AH} \cdots \mathrm{B}$ fragment. ${ }^{5}$ In the simplest model, the properties of the $\mathrm{AH} \cdots \mathrm{B}$ fragment depend on two variables - the $\mathrm{AH}$ and $\mathrm{A} \cdots \mathrm{B}$ interatomic distances. In a more adequate model, a third variable must be considered, which corresponds to the bending angle.

In spite of its underlying approximation, the $\mathrm{OH} \cdots \mathrm{O}$ 
fragment model has been used.successfully to examine a number of important distinctive features of the $\mathrm{H}$-bonded systems containing the $\mathrm{OH} \cdots \mathrm{O}^{5,9-13}$ and $\mathrm{AH} \cdots \mathrm{B}$ fragments. ${ }^{14,15}$ This approach is particularly attractive in that one can apply the adiabatic (Stepanov) separation of the "fast" (H atom) and "slow" (heavy atoms) subsystems, ${ }^{9}$ making the results easily interpretable in terms of simple physical concepts.

\section{Hamiltonian}

Consider the $\mathrm{H}$-bonded triatomic fragment $\mathrm{A}-\mathrm{H} \cdots \mathrm{B}$, with masses $M_{\mathrm{A}}, M_{\mathrm{H}}$, and $M_{\mathrm{B}}$, where $M_{\mathrm{A}}\left(M_{\mathrm{B}}\right) \gg M_{\mathrm{H}}$. The $\mathrm{A}-\mathrm{H}$ and $\mathrm{A} \cdots \mathrm{B}$ interatomic distances are designated respectively as $r$ and $R$, and the $\mathrm{B} \cdots \mathrm{A}-\mathrm{H}$ angle by $\theta$. The centerof-mass translation has no physical significance for our model and is ignored. The relative kinetic energy has a separable form using Jacobi coordinates. Let us assume that one coordinate vector $\mathbf{r}$ connects the $\mathrm{A}$ and $\mathrm{H}$ atoms and the other, $\mathbf{R}^{\mathrm{Jac}}$ gives the position of the B atom with respect to the center of mass of the AH pair. Because $M_{\mathrm{A}}\left(M_{\mathrm{B}}\right) \gg M_{\mathrm{H}}$, the $\mathbf{R}^{\mathrm{Jac}}$ variable can be replaced by $\mathbf{R}^{16}$

Following Ref. 17, we suppress description of the rotational degrees of freedom associated with the AHB fragment. In the $(\mathbf{r}, \mathbf{R})$ coordinates the vibrational Schrödinger equation becomes

$$
\left\{-\frac{\hbar^{2}}{2 m^{*}} \frac{\partial^{2}}{\partial \mathbf{r}^{2}}-\frac{\hbar^{2}}{2 M} \frac{\partial^{2}}{\partial \mathbf{R}^{2}}+\operatorname{PES}(\mathbf{r}, \mathbf{R})-E\right\} \Psi(\mathbf{r}, \mathbf{R})=0
$$

where the reduced masses are

$$
m^{*}=M_{\mathrm{H}} M_{\mathrm{A}} /\left(M_{\mathrm{H}}+M_{\mathrm{A}}\right)
$$

for the proton motion, and

$$
M=M_{\mathrm{B}}\left(M_{\mathrm{A}}+M_{\mathrm{H}}\right) /\left(M_{\mathrm{B}}+M_{\mathrm{A}}+M_{\mathrm{H}}\right)
$$

for the heavy particles.

Solution of Eq. (1), subject to appropriate boundary conditions, yields the vibrational energy levels $\left\{E_{j}\right\}$ and corresponding eigenfunctions $\left\{\Psi_{j}(\mathbf{r}, \mathbf{R})\right\}$. Replacing $\mathbf{r}$ by its representation in polar coordinates $(r, \theta)$, where $\theta$ is the angle between $\mathbf{r}$ and $\mathbf{R}$, the simplified Hamiltonian can be written as

$$
\begin{aligned}
H= & -\frac{\hbar^{2}}{2 m^{*}} \frac{\partial^{2}}{\partial r^{2}}-\frac{\hbar^{2}}{2 m^{*} r^{2}} \frac{\partial^{2}}{\partial \theta^{2}}-\frac{\hbar^{2}}{2 M} \frac{\partial^{2}}{\partial R^{2}} \\
& +\operatorname{PES}(r, \theta, R),
\end{aligned}
$$

where $\operatorname{PES}(r, \theta, R)$ is a three-dimensional potential energy surface in a given electronic state. [Strictly speaking, one should use the variable $\theta^{\text {Jac16 }}$ in Eq. (3) rather than $\theta$; however, since $\theta^{\text {yac }} \sim \theta$, little accuracy is lost by use of the latter.]

The wave equation with Hamiltonian (3) is solved using the following scheme:

(1) apply the adiabatic approximation with respect to deformation mode (variable $\theta$ ) - the eigenvalues of this mode are defined as functions of $r$ and $R$;
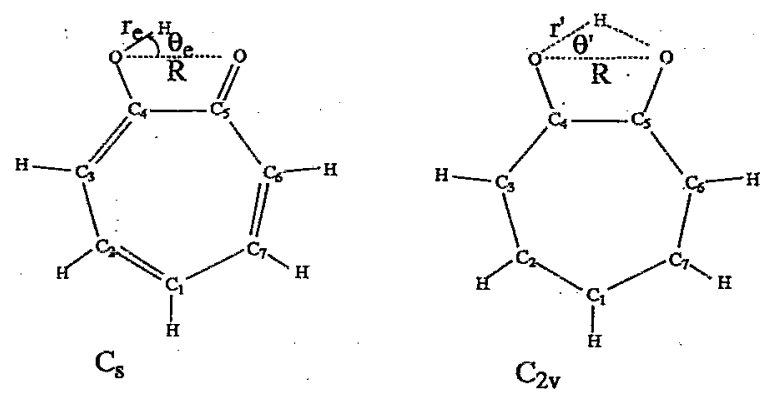

FIG. 1. Numbering scheme for atoms and geometric parameters of the $\mathrm{O}-\mathrm{H} \cdots \mathrm{O}$ fragment for the equilibrium $\left(C_{s}\right)$ and symmetric $\left(C_{2 v}\right)$ structures of tropolone.

(2) assume adiabatic approximation with respect to the $\mathrm{H}$ stretching mode (variable $r$ ) -the eigenvalues of this mode are defined as functions of $R$;

(3) solve $O \cdots O$ stretch dynamics (variable $R$ ) explicitly.

\section{Adiabatic description}

Using adiabatic separation of the $\theta, r$, and $R$ variables, one can write the complete nuclear wave function [see $\mathrm{Eq}$. (1)] in the form

$$
\Psi(\mathbf{r}, \mathbf{R}) \sim \Phi_{m v n}(\theta, r, R)=\zeta_{m}(\theta, r, R) \phi_{m v}(r, R) \chi_{m v n}(R),
$$

where $m, v$, and $n$ are the vibrational quantum numbers corresponding to the $\theta, r$, and $R$ modes, respectively. The applicability of the adiabatic separation of vibrational coordinates has been convincingly demonstrated in a series of papers. ${ }^{9,13,17-26}$

It is convenient to define a new variable $q=r \theta$, where $r$ is held fixed. The PES is then constructed within the new set of coordinates $q, r, R$. Profiles of the PES for fixed $r$ and $R$, $P(q, r, R)$, are approximated by polynomials in $q$, with $r$ and $R$ as parameters. Taking into account that $r$ is fixed for a given set of $q$, the corresponding wave equation for the deformation vibration can be written as

$$
H_{1} \zeta_{m}(q, r, R)=U_{m}(r, R) \zeta_{m}(q, r, R),
$$

where

$$
H_{1}=-\frac{\hbar^{2}}{2 m^{*}} \frac{\partial^{2}}{\partial q^{2}}+P(q, r, R)
$$

and $m^{*}$ is defined by Eq. (2a). Solution of Eq. (5) yields a family of two-dimensional (2-D) PESs, $U_{m}(r, R)$.

In order to translate to a "standard" 2-D PES for the symmetric OHO fragment (see Figs. 2 in Refs. 10 and 13), in which the origin of the $\mathrm{H}$ (D) stretching coordinate is the center of symmetry of the OHO (ODO) fragment, the $r$ coordinate can be replaced by symmetry-adapted internal coordinate $x$ as follows: $x=r^{\prime}-r$, where $r^{\prime}$ is the value of $r$ in the symmetric $C_{2 v}$ structure at given $R$, see Fig. 1 . (The $r^{\prime}$ value varies with $R$ ). The profiles of these surfaces at fixed $R$ 
are approximated by appropriate functions of $x$, which depend on $R$ parametrically, see Appendix A. The corresponding wave equation for $H$ (D) stretching is

$$
H_{2} \phi_{m v}(x, R)=\epsilon_{m v}(R) \phi_{m v}(x, R),
$$

where the Hamiltonian is

$$
H_{2}=-\frac{\hbar^{2}}{2 m^{*}} \frac{\partial^{2}}{\partial x^{2}}+U_{m}(x, R)
$$

The result is a family of adiabatic curves $\epsilon_{m v}(R)$ which describe the potential energy of the $\mathrm{O} \cdots \mathrm{O}$ stretching vibrations as a function of $R$. As for the aforementioned profiles, $\epsilon_{m v}(R)$ can also be approximated by polynomials.

The wave equation that remains to be solved for $\mathrm{O} \cdots \mathrm{O}$ stretching vibrations is

$$
H_{3} \chi_{m v n}(R)=E_{m v n} \chi_{m v n}(R),
$$

where the Hamiltonian is

$$
H_{3}=-\frac{\hbar^{2}}{2 M} \frac{\partial^{2}}{\partial R^{2}}+\epsilon_{m v}(R) .
$$

and $M$ is defined by Eq. ( $2 b)$. The applicability of the Hamiltonians (6) and (7) for $\mathrm{OH} \cdots \mathrm{O}$ fragment dynamics is verified in Appendix B.

\section{Potential energy surface and dynamics calculations}

$A b$ initio methods at the SCF and CIS ${ }^{27}$ levels häve been used to generate the potential energy surfaces of the $S_{0}$ and $S_{1}$ electronic states, respectively. The computations were performed using the GAUSSIAN 92 package. ${ }^{28}$ The basis set was based upon the $6-31 G^{29}$ type: $p$ type polarization functions $(\alpha=1.10)$ were added to the bridging $\mathrm{H}$ and $d$-type functions $(\alpha=0.80)$ to $O$. After choosing specific values of $r$, $\theta$, and $R$, within a three-dimensional grid, the remaining geometrical degrees of freedom of the entire molecule were all optimized. Vibrational eigenstates were obtained using numerical methods described in Ref. 30 [Eqs. (5) and (6)] and renormalized Numerov method ${ }^{31}$ [Eq. (7)].

\section{RESULTS}

\section{Geometries and energetics}

The equilibrium and saddle point geometries were fully optimized at the SCF $\left(S_{0}\right)$ and CIS $\left(S_{1}\right)$ levels of theory. The details of these geometries are presented in Table I, together with the available experimental data, using atomic labeling defined in Fig. 1. The geometric parameters for the ground state, obtained here with the partially polarized basis. set, are quite similar to the fully polarized $6-31 G^{* *}$ geometries. $^{32}$ Comparison of the first two columns of data in Table I illustrates that agreement with the experimental crystal structure is also good. Of greatest import here are the aspects of the $\mathrm{H}$-bond geometry. The interoxygen distance is reproduced well; of particular accuracy is the nonlinearity of the $\mathrm{H}$ bond, which is correct to within less than one degree.

The $S_{1}$ state arises from a $\pi \rightarrow \pi^{*}$ transition, in accord with experimental findings. ${ }^{34}$ Also in agreement with experiment, ${ }^{35}$ calculations reveal a small contraction of the $\mathrm{O} \cdots \mathrm{O}$ equilibrium distance accompanies this excitation. As one progresses around the ring, the single bonds become shorter and the double bonds longer in $S_{1}$ as compared to $S_{0}$. Of particular interest because of its proximity to the $\mathrm{H}$ bond, the $\mathrm{C}_{4} \mathrm{C}_{5}$ bond is lengthened by $\sim 0.035 \AA$, compared to the experimental estimate of $0.024 \AA^{34}$

Calculated rotational constants are listed in Table II, together with experimental data. The values for the $S_{0}$ state resemble those obtained using the fully polarized $6-31 G^{* *}$ basis set, ${ }^{32}$ and are $1 \%-2 \%$ larger than values used in Ref. 34 for analysis of the profile of the $370 \mathrm{~nm}$ uv absorption band of tropolone. The theoretical rotational constants for the $S_{1}$ state agree with experimental data fairly well, as do the changes arising from electronic excitation.

The fully optimized geometry computed for the symmetrical transition state (TS) is listed on the right side of Table I. The character of this $C_{2 v}$ structure as a true transition state in the $S_{0}$ state was verified by the presence of a single imaginary vibrational eigenvalue; it was found to be equal to $1927 \mathrm{~cm}^{-1}$, in accord with previous ab initio data. ${ }^{32}$ The shortening of the distance between the two oxygens in the TS structure in comparison with the equilibrium geometry is evident in both the ground and excited states. This contraction amounts to roughly $0.25 \AA$. The transition state also finds the proton closer to the $\mathrm{O} \cdots \mathrm{O}$ axis, with a $\sim 20^{\circ}$ reduction in $\theta(\mathrm{OOH})$.

The calculated total energies from this work are also reported in Table I. The "relaxed" barrier height, computed as the difference between the energies of the TS and equilibrium geometry is equal to $15.7 \mathrm{kcal} / \mathrm{mol}$ for the $S_{0}$ state. It is very close to $16 \mathrm{kcal} / \mathrm{mol}$ estimated in Ref. 35 from fitting IR frequencies, tunnel splittings, and H/D isotope effects to $2 \mathrm{D}$ PES. These barriers are much higher than some current estimates of $6-7 \mathrm{kcal} / \mathrm{mol}$ for malonaldehyde ${ }^{2,3}$ and ca. $6 \mathrm{kcal} /$ mol for the correlated relaxed barrier in tropolone. ${ }^{36}$ Since the $\sim 21 \mathrm{~cm}^{-1}$ ground state tunnel splitting observed for malonaldehyde ${ }^{37}$ is ca. 21 times more than that reported for tropolone $\left(0.99 \mathrm{~cm}^{-16,38}\right)$, the $\sim 15.7 \mathrm{kcal} / \mathrm{mol}$ "relaxed" barrier for tropolone in the ground electronic state seems reasonable.

The relaxed barrier height of the excited state was calculated to be $12.4 \mathrm{kcal} / \mathrm{mol}$ using the CIS method, lower by $3.3 \mathrm{kcal} / \mathrm{mol}$ than the ground state barrier. This excited state barrier in tropolone is higher than the analogous estimate of 6-7 $\mathrm{kcal} / \mathrm{mol}$ for malonaldehyde., ${ }^{2,3}$ On the other hand, since the $\sim 21 \mathrm{~cm}^{-1}$ ground state tunneling splitting observed for malonaldehyde $\mathrm{d}^{37}$ is very close to the $\sim 20 \mathrm{~cm}^{-1}$ for tropolone in its $S_{1}$ state, $^{34}$ the $.12 .4 \mathrm{kcal} / \mathrm{mol}$ relaxed barrier for tropolone may represent an overestimate.

The dynamics calculations below make use of the PES obtained at the SCF (CIS) level for the ground (excited) state. It should also be stressed that the goal of this work is a detailed understanding of the interactions between the various nuclear motions, which requires knowledge of an extensive region of the PES. The uncorrelated surface is expected to provide a reasonable first approximation to the character of this surface, particularly since geometry optimizations have been performed at each point.

Because of the use of the adiabatic approximation, we will discuss below barriers obtained for a given fixed 
in.

TABLE I. Bond lengths, angles and the total energies of the equilibrium $\left(C_{s}\right)$ and transition state $\left(C_{2 v}\right)$ structures of tropolone in the $S_{0}$ and $S_{1}$ states.

\begin{tabular}{|c|c|c|c|c|c|}
\hline \multirow[t]{2}{*}{ Bond lengths, $\AA$} & \multicolumn{3}{|c|}{$C_{s}$ structure } & \multicolumn{2}{|c|}{$C_{2 v}$ structure } \\
\hline & $s_{0}$ & crystal $^{2}$ & $s_{\mathrm{t}}$ & $s_{0}$ & $S_{1}$ \\
\hline $0 \cdots 0$ & 2.529 & 2.553 & 2.523 & 2.254 & 2.271 \\
\hline $\mathrm{O}-\mathrm{H}$ & 0.953 & 0.94 & 0.956 & 1.206 & 1.213 \\
\hline $\mathrm{C}_{5}=\mathrm{O}$ & 1.222 & 1.261 & 1.230 & 1.275 & 1.270 \\
\hline $\mathrm{C}_{4}-\mathrm{O}$ & 1.342 & 1.330 & 1.330 & 1.275 & 1.270 \\
\hline $\mathrm{C}_{1} \mathrm{C}_{2}$ & 1.351 & 1.341 & 1.424 & 1.388 & 1.404 \\
\hline $\mathrm{C}_{2} \mathrm{C}_{3}$ & 1.427 & 1.393 & 1.388 & 1.387 & 1.404 \\
\hline $\mathrm{C}_{3} \mathrm{C}_{4}$ & 1.349 & 1.379 & 1.387 & 1.386 & 1.387 \\
\hline $\mathrm{C}_{4} \mathrm{C}_{5}$. & 1.482 & 1.454 & 1.517 & 1.485 & 1.557 \\
\hline $\mathrm{C}_{5} \mathrm{C}_{6}$ & 1.446 & 1.410 & 1.419 & 1.386 & 1.387 \\
\hline $\mathrm{C}_{6} \mathrm{C}_{7}$ & 1.350 & 1.373 & 1.403 & 1.387 & 1.404 \\
\hline $\mathrm{C}_{1} \mathrm{C}_{7}$ & 1.429 & 1.410 & 1.385 & 1.388 & 1.404 \\
\hline $\mathrm{C}_{1} \mathrm{H}$ & 1.073 & 1.07 & 1.074 & 1.073 & 1.075 \\
\hline $\mathrm{C}_{2} \mathrm{H}$ & 1.074 & 1.04 & 1.073 & 1.075 & 1.073 \\
\hline $\mathrm{C}_{3} \mathrm{H}$ & 1.073 & 1.09 & 1.074 & 1.072 & 1.073 \\
\hline $\mathrm{C}_{6} \mathrm{H}$ & 1.073 & 1.09 & 1.072 & 1.072 & 1.073 \\
\hline $\mathrm{C}_{7} \mathrm{H}$ & 1.075 & 1.08 & 1.074 & 1.075 & 1.073 \\
\hline \multicolumn{6}{|l|}{ Bond angles, deg } \\
\hline OO-H & 42.6 & 43 & 42.7 & 20.9 & 20.5 \\
\hline $\mathrm{C}_{1} \mathrm{C}_{2} \mathrm{C}_{3}$ & 129.4 & 129.9 & 128.5 & 130.6 & 129.2 \\
\hline $\mathrm{C}_{2} \mathrm{C}_{3} \mathrm{C}_{4}$ & 129.7 & 129.4 & 129.0 & 127.8 & 127.1 \\
\hline $\mathrm{C}_{3} \mathrm{C}_{4} \mathrm{C}_{5}$ & 129.5 & 128.8 & 129.0 & 127.8 & 128.2 \\
\hline $\mathrm{C}_{4} \mathrm{C}_{5} \mathrm{C}_{6}$ & 123.4 & 124.2 & 125.4 & 127.8 & 128.2 \\
\hline $\mathrm{C}_{5} \mathrm{C}_{6} \mathrm{C}_{7}$ & 130.3 & 130.6 & 128.7 & 127.8 & 127.1 \\
\hline $\mathrm{C}_{6} \mathrm{C}_{7} \mathrm{C}_{1}$ & 130.4 & 129.4 & 129.9 & 130.6 & 129.2 \\
\hline $\mathrm{C}_{7} \mathrm{C}_{1} \mathrm{C}_{2}$ & 127.2 & 127.5 & 129.6 & 127.7 & .131 .1 \\
\hline $\mathrm{C}_{5} \mathrm{C}_{4} \mathrm{O}_{8}$ & 112.3 & 114.7 & 113.6 & 107.6 & 106.3 \\
\hline $\mathrm{C}_{4} \mathrm{C}_{5} \mathrm{O}_{9}$ & 115.7 & 115.4 & 112.6 & 107.6 & 106.3 \\
\hline $\mathrm{C}_{2} \mathrm{C}_{1} \mathrm{H}$ & 117.2 & 118 & 114.7 & 116.2 & 114.5 \\
\hline $\mathrm{C}_{1} \mathrm{C}_{2} \mathrm{H}$ & 116.5 & 116 & 115.6 & 115.0 & 115.4 \\
\hline $\mathrm{C}_{2} \mathrm{C}_{3} \mathrm{H}$ & 115.8 & 115 & 117.0 & 117.5 & 117.8 \\
\hline $\mathrm{C}_{7} \mathrm{C}_{6} \mathrm{H}$ & 117.9 & 115 & 118.0 & 117.5 & 117.8 \\
\hline $\mathrm{C}_{1} \mathrm{C}_{7} \mathrm{H}$ & 114.4 & 116 & 115.4 & 115.0 & 115.4 \\
\hline $\mathrm{C}_{4} \mathrm{OH}$ & 107.0 & 107 & 107.2 & 93.3 & 94.2 \\
\hline Energy, a.u. & -418.148765 & & -417.983879 & -418.123720 & -417.964178 \\
\hline
\end{tabular}

${ }^{\mathrm{a}}$ Dimerized monomer from crystal, Ref. 33.

$R(\mathrm{O} \cdots \mathrm{O})$, termed "frozen" barriers, so as to distinguish them from the relaxed barrier.

\section{Adiabatic proton (deuteron) states}

Each section of the PES $P(q, r, R)$ has only a single minimum when both $r$ and $R$ are held equal to constant values; this minimum corresponds to the equilibrium $\theta_{e}\left(q_{e}=r \theta_{e}\right)$ at given $r$ and $R$. The $P$ function was approximated by a polynomial of second order:

$$
P(q, r, R)=c(r, R)+c_{1}(r, R) q+c_{2}(r, R) q^{2} .
$$

TABLE II. Calculated and experimental rotational constants for the $S_{0}$ state and changes arising from the $S_{0} \rightarrow S_{1}$ transition (all values in $\mathrm{GHz}$ ).

\begin{tabular}{lcccccc}
\hline \hline & $A$ & $B$ & $C$ & $\Delta A$ & $\Delta B$ & $\Delta C$ \\
\hline Calc. $^{\mathrm{a}}$ & 2.7551 & 1.6723 & 1.0406 & -0.0758 & 0.0054 & -0.0089 \\
Expt. $^{\mathrm{a}}$ & 2.7300 & 1.6433 & 1.0258 & -0.0502 & -0.0032 & -0.0084 \\
\hline \hline
\end{tabular}

${ }^{\mathrm{a}}$ Reference 34
It was found that $c_{1}^{2}(r, R) \ll 4 c_{2}(r, R)$ so the eigenvalues of the bending Schrödinger equation are those of a harmonic oscillator

$$
U_{m}(r, R)=\hbar \omega(r, R)(m+1 / 2)+c(r, R),
$$

where $\omega(r, R)=\left\{2 c_{2}(r, R) / m^{*}\right\}^{1 / 2}$.

One can see from Eq. (9) that $U_{m}(r, R)$ is a sum of bending vibrations $\hbar \omega(m+1 / 2)$ and potential energy of $\mathrm{O} \cdots \mathrm{O}$ and $\mathrm{O}-\mathrm{H}$ stretching vibrations, designated as $c(r, R)$.

The magnitude of the zero-point vibrational energy correction to the frozen barrier height at the equilibrium $\mathrm{O} \cdots \mathrm{O}$ distance, caused by the $\mathrm{OH}$ bending, is equal to $+0.32 \mathrm{kcal} /$ $\mathrm{mol}\left(120 \mathrm{~cm}^{-1}\right)$ in the $S_{0}$ state and $+0.44 \mathrm{kcal} / \mathrm{mol}(150$ $\left.\mathrm{cm}^{-1}\right)$ in $S_{1}$. These values are two orders of magnitude lower than the barrier itself $\left(29.9\right.$ and $24.0 \mathrm{kcal} / \mathrm{mol}$ in the $S_{0}$ and $S_{1}$ states, respectively). This result suggests that deformation vibrations play only a minor role in the modulation of effective potential barrier of PT in TRN in either electronic state.

As a result of solving Eq. (5) we obtain adiabatic surfaces $U_{m}(r, R)$ for each quantum number $m$ for each isotope 


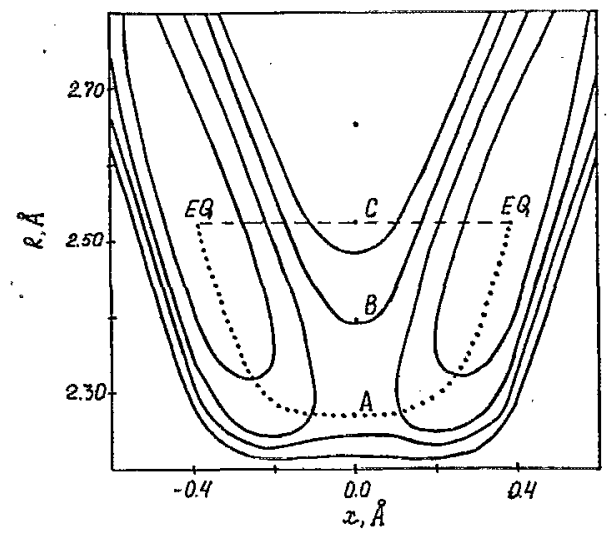

FIG. 2. Adiabatic potential energy surface $U_{0}^{*}(x, R)$ for proton transfer in the $S_{1}$ state. The dotted curve corresponds to the minimum energy path. Contours are in increments of $5 \mathrm{kcal} / \mathrm{mol}$. The points $A, B, C$ all lie along the $x=0$ axis and EQ represents the equilibrium geometry, with relative energy zero.

in a given electronic state. In order to be consistent with the symmetry of the $\mathrm{OH}$ stretch problem, the $r$ coordinate was replaced by $x=r^{\prime}-r$, where $r^{\prime}$ is the value of the $r(\mathrm{OH})$ distance in the symmetric $C_{2 v}$ structure at a given $R$ (see Fig. 1). This transformation places the origin of the "fast" subsystem at the $\mathrm{O} \cdots \mathrm{O}$ midpoint. The proton potential $U_{0}^{*}(x, R)$ (* indicates the excited $S_{1}$ state) is displayed in two-dimensional form in Fig. 2. Note that contours are not displayed for regions of the PES where the energy exceeds that of the equilibrium structure by more than $20 \mathrm{kcal} / \mathrm{mol}$. The latter (EQ) is characterized by a longer $\mathrm{O} \cdots \mathrm{O}$ distance than the TS structure $(A)$, for which $x=0$. The minimum energy path through the PES is indicated by the dotted curve which passes through different values of $R$.

The frozen barrier at any given value of $R$ is associated with a horizontal slice through the PES. Each slice through the PES at a fixed value of $R$ was approximated by a function, suggested by some of the authors earlier ${ }^{11,13}$ (see Appendix A). After solution of the Schrödinger equation for proton (deuteron) stretch one obtains a family of adiabatic energy levels which are functions of $R: \epsilon_{m v}(R)$. The first quantum number $m$ refers to deformation vibrations while the second, $v$, corresponds to the OH (OD) stretch. These levels are shown in Fig. 3 within the energy profiles at each fixed $R$. Because of the longer $R(\mathrm{OO})$ distance in the equilibrium than TS geometry, the barrier at the equilibrium $0 \cdots$ distance is rather high, e.g., $24 \mathrm{kcal} / \mathrm{mol}$ in $S_{1}$, see Fig. 3(c). Hence, at this $R$, the $\mathrm{H}$ (D) vibrational levels occur in closely spaced pairs (doublets), with separations not discernible in the scale of the figure. The energy splitting between levels in a given doublet is very much smaller than the energy gap between doublets. At shorter $\mathrm{O} \cdots \mathrm{O}$ distances the splitting between levels in the doublets becomes larger, but $\epsilon_{01}-\epsilon_{00}$ remains much smaller than the energy gap between $\epsilon_{01}$ and the next excited level, i.e., $\epsilon_{01}-\epsilon_{00} \ll \epsilon_{02}-\epsilon_{01}$, see Figs. 3(a) and 3(b).
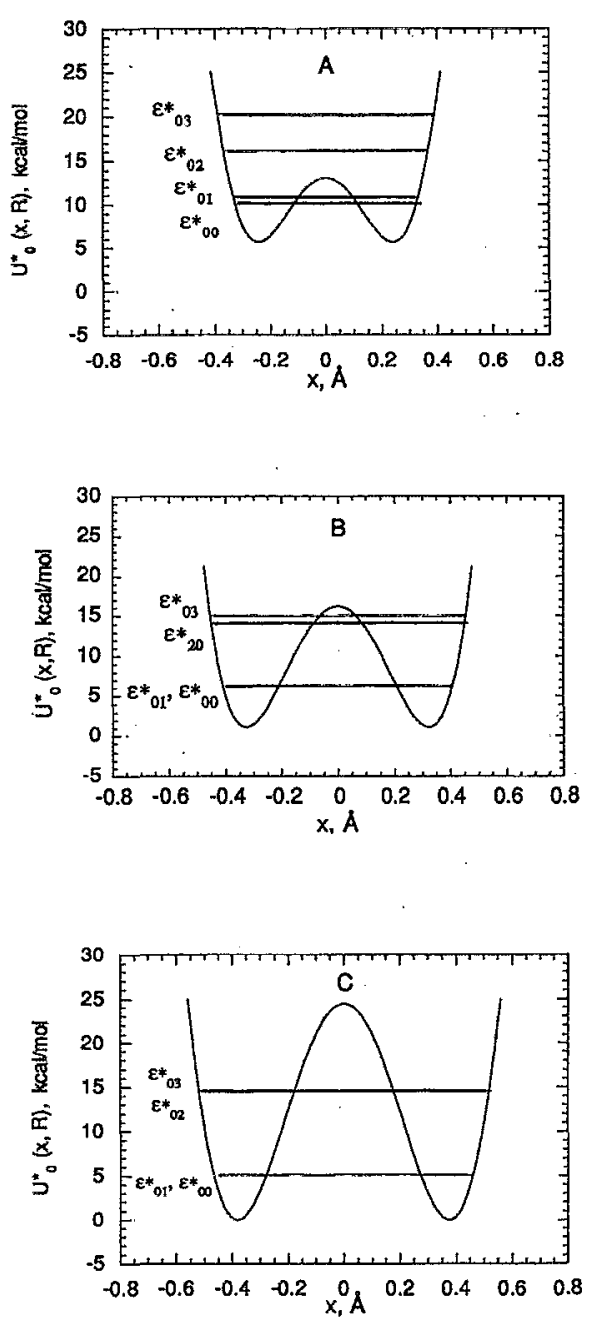

FIG. 3. Slices through the adiabatic proton potential energy surface $U_{0}^{*}(x, R)$ for three fixed $R$ values, (a) 2.271 , (b) 2.397 , (c) $2.523 \AA$. The points $A, B$, and $C$ from Fig. 2 correspond to the tops of the respective barriers. The four lowest lying proton stretch levels are shown but are not all apparent due to small separation in certain cases.

\section{Ground and excited state splitting}

The $\epsilon_{m v}(R)$ functions were approximated by polynomials and the Schrödinger equation (7) solved for $O \cdots O$ stretching vibrations, leading to eigenvalues $E_{m u n}$ depending on three quantum numbers, see Fig. 4. It was mentioned above that the proton (deuteron) stretch levels with quantum numbers $v=0$ and 1 occur in closely-spaced pairs (doublets); hence each $\mathrm{O} \cdots \mathrm{O}$ stretch and $\mathrm{H}$ (D) bend level will exist as a doublet. The energy difference

$$
\Delta_{m v n}=E_{m, v+1, n}-E_{m v n}
$$

represents the tunneling splitting of any given state, $m v n$ (where $v$ is an even number). In this terminology, the ground-state splitting

$$
\Delta_{000}=E_{010}-E_{000}
$$

and that of the first excited level of the OH (OD) stretch is

$$
\Delta_{020}=E_{030}-E_{020} \text {. }
$$




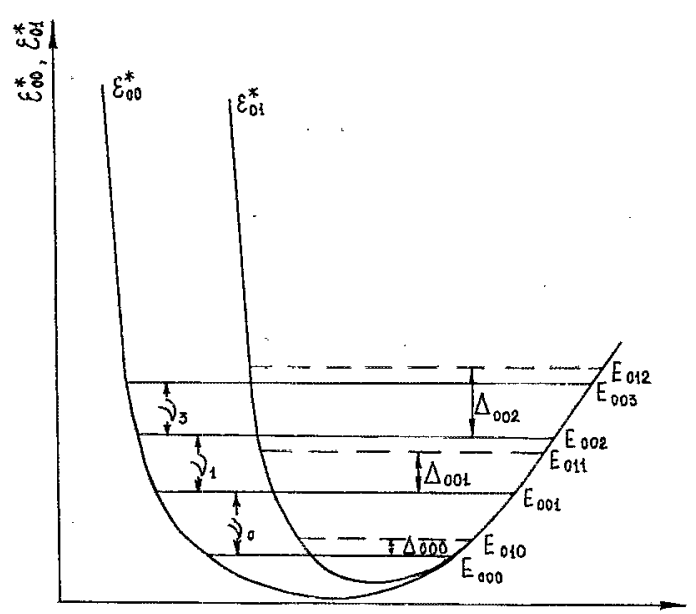

FIG. 4. Schematic diagram of the $\epsilon_{00}^{*}$ and $\epsilon_{01}^{*}$ energies as functions of interoxygen separation. Energy levels are not drawn to scale. Solid lines refer to $\epsilon_{00}^{*}$ and broken lines to $\epsilon_{01}^{*}$.

The $\Delta_{000}$ quantity may be abbreviated as $\Delta_{0}$, suppressing the zero $m$ and $n$ indices. Similarly, $\Delta_{m 00}=E_{m 10}-E_{m 00}$ refers to the proton stretch tunnel splitting with the $m$ th bending level excited and $\Delta_{00 n}=E_{01 n}-E_{00 n}$ to the proton level tunnel splitting with the $\mathrm{O} \cdots \mathrm{O}$ level excited, see Fig. 4.

The fundamental of the $\mathrm{O} \cdots \mathrm{O}$ stretch vibration can analogously be associated with the energy difference

$$
\nu_{0} \equiv \nu_{000}=E_{001}-E_{000}
$$

and $\delta$ is used to indicate the change in bending quantum number $m$ where

$$
\delta_{0} \equiv \delta_{000}=E_{100}-E_{000}
$$

corresponds to the fundamental frequency of bending vibrations. The frequency of the $\mathrm{OH}(\mathrm{OD})$ stretch corresponds to a change in $v$, starting from an odd number.

$$
\nu(\mathrm{OH})=E_{020}-E_{010} \sim E_{030}-E_{000} \text {. }
$$

Energy levels $E_{m u n}$ calculated using Eq. (7) for the $S_{0}$ state are reported in Table III, along with the splitting. Rela-

TABLE III. Calculated values of the relative energies and tunnel splittings

\begin{tabular}{|c|c|c|c|c|c|c|}
\hline \multicolumn{3}{|c|}{ Quantum nos. } & \multicolumn{2}{|c|}{ Relative energy, $E_{m v n}$} & \multicolumn{2}{|c|}{ Tunnel splitting, $\Delta_{m v n}{ }^{a}$} \\
\hline$m$ & $v$ & $n$ & $\mathrm{H}$ & $\mathrm{D}$ & $\mathrm{H}$ & D \\
\hline 0 & 0 & 0 & 0 & 0 & $0.9\left(0.99^{b}\right)$ & $<0.1\left(<0.17^{\circ}\right)$ \\
\hline 0 & 0 & 1 & $450\left(360^{d}\right)$ & $426\left(338^{\mathrm{e}}\right)$ & 3.0 & $\sim 0.1$ \\
\hline 0 & 0 & 2 & 891 & 862 & 7.2 & 0.4 \\
\hline 0 & 0 & 3 & 1326 & 1299 & 13.0 & 1.2 \\
\hline 1 & 0 & 0 & $1381\left(1450^{f}\right)$ & $988\left(1103^{f}\right)$ & 0.6 & $<0.1$ \\
\hline 0 & 2 & 0 & $3570\left(3121^{f}\right)$ & $2677\left(2326^{f}\right)$ & 81.7 & $4.1\left(15.6^{g}\right)$ \\
\hline
\end{tabular}
in the $S_{0}$ state of TRNOH and TRNOD. Available experimental data are given in parentheses. All values in $\mathrm{cm}^{-1}$.

"See Eqs. (10)-(11).

'References 6 and 38 .

'Reference 34.

dFrom Eq. (12), expt data from Ref. 39.

EFrom Eq. (12), expt data from Ref. 40.

Reference 41.

${ }^{\text {g}}$ Estimated in Ref. 35 from data in neon matrix. tively low precision of the tunnel splittings in Tables III-V is caused by the polynomial approximation of the adiabatic curves $E_{m v}(R)$. Calculated values agree rather well with the available experimental data on the tunnel splitting and vibration frequencies of TRN and its deuterated form. The absolute values of the calculated $\mathrm{OH}(\mathrm{OD})$ stretching and bending frequencies differ from experiment by less than $20 \%$. It should be noted that our treatment of $O H$ (OD) stretch provides sufficiently good agreement with experiment, by several hundred $\mathrm{cm}^{-1}$, compared to a harmonic approximation of the entire molecule, using a 6-31G basis set, ${ }^{32}$ which yields a very high value of $3923 \mathrm{~cm}^{-1}$. In accord with experiment, ${ }^{40,41}$ the $\mathrm{O} \cdots \mathrm{O}$ stretching vibrational frequency for $\mathrm{H}\left(450 \mathrm{~cm}^{-1}\right)$ is calculated to be a little higher than for $\mathrm{D}$, while these absolute values are ca. $25 \%$ larger than experiment. The ground state tunnel splitting for OHO of $0.9 \mathrm{~cm}^{-1}$ agrees nicely with experimental data, ${ }^{6,38}$ while the ground state splitting of the ODO fragment is quite a bit smaller than for $\mathrm{OHO}$, in conformity with the conclusions of Tomioka et $a .^{42}$ It is important to stress that the tunnel splitting of the proton stretch level for excited $\mathrm{O} \cdots \mathrm{O}$ vibrations $\left(\Delta_{00 n}\right)$ depends strongly on $n$ (Tables III and IV) in accord with results of other authors. ${ }^{4,10}$ The tunnel splitting of the second pair of $\mathrm{OH}$ (OD) vibrational states $\Delta_{020}$ was found to be equal to 81.7 and $4.1 \mathrm{~cm}^{-1}$, respectively. The latter value is comparable to the tunnel splitting of the tropolone OD stretch (15.6 $\mathrm{cm}^{-1}$ ) estimated by Redington ${ }^{35}$ from data in neon matrix. (The corresponding splitting for the $\mathrm{OH}$ stretch was not reported.) In contrast to the $\mathrm{O} \cdots \mathrm{O}$ and $\mathrm{OH}$ stretch, the excitation of $\mathrm{OH}(\mathrm{OD})$ bending vibrations has little effect on the tunnel splitting, witnessed by the similarity of the splitting quantities listed in the first, fifth, and sixth rows of Table III. We estimate an error which is introduced by neglect of the dependence of the bending energy on $r$ and $R$, i.e., by omission of the first term on the right side of Eq. (9). The estimated value of $\Delta_{0}(\mathrm{H})$ was found to be only ca. $8 \%$ larger than the "exact" ones.

These results lead to two important conclusions: (1) the $\mathrm{O} \cdot \cdot \mathrm{O}$ vibrations are strongly coupled with $\mathrm{PT}$ motion causing an increase of the proton stretching level splitting; (2) the deformation vibrations are coupled with PT motion, but weakly.

An inspection of the spacing of the $\mathrm{O} \cdots \mathrm{O}$ levels in Table III shows that the vibrations at $m=0$ and $v=0$ can be treated approximately as harmonic in the $S_{0}$ state. ${ }^{6}$ In summary, the model which makes use of a localized $\mathrm{OH} \cdots \mathrm{O}$ fragment, combined with $a b$ initio SCF calculations, provides a quantitatively correct picture of hydrogen bonding and proton transfer in the $S_{0}$ state of tropolone.

Analogous calculated data for the $S_{1}$ state may be seen in Table IV to reproduce the principal experimental trends:

(1) An increase in the ground vibrational state tunnel splitting for both isotopic forms ${ }^{34}$ occurs as a result of the electronic excitation. This increase factor is calculated to be $(3: 1 / 0.9)=3.5$, as compared to an experimental ratio of $(19.92 / 0.99)=20$.

(2) As in the $S_{0}$ state, the tunneling splitting becomes larger as the $\mathrm{O} \cdots \mathrm{O}$ stretch is excited ${ }^{40,43}$ in $S_{1}$. 
TABLE IV. Calculated values of the relative energies and tunnel splittings in the $S_{1}$ state of TRNOH and TRNOD. Available experimental data are given in parentheses. All values in $\mathrm{cm}^{-1}$.

\begin{tabular}{|c|c|c|c|c|c|c|}
\hline \multicolumn{3}{|c|}{ Quantum Nos. } & \multicolumn{2}{|c|}{ Relative energy, $E_{m v n}$} & \multicolumn{2}{|c|}{ Tunnel splitting, $\Delta_{m v n}{ }^{\mathrm{a}}$} \\
\hline$m$ & $v$ & $n$ & $\mathrm{H}$ & D & $\mathrm{H}$ & $\mathrm{D}$ \\
\hline 0 & 0 & 0 & 0 & 0 & $3.1\left(19.9^{d}\right)$ & $0.1\left(2.0^{\mathrm{e}}\right)$ \\
\hline 0 & 0 & 1 & $425\left(302^{b}\right)$ & $423\left(299^{c}\right)$ & $8.9\left(31.0^{d}\right)$ & $0.8\left(11.0^{\mathrm{e}}\right)$ \\
\hline 0 & 0 & 2 & 854 & 856 & $18.9\left(29.0^{d}\right)$ & $2.1\left(13.0^{\mathrm{e}}\right)$ \\
\hline 0 & 0 & 3 & 1283 & 1291 & 33.5 & 5.1 \\
\hline 1 & 0 & 0 & 1329 & 948 & 2.5 & 0.1 \\
\hline 0 & 2 & 0 & 3127 & 2501 & 204.3 & 10.8 \\
\hline
\end{tabular}

asee Eqs. (10)-(11).

'From Eq (12), expt data from Ref. 6.

'From Eq (12), expt data from Ref. 40.

${ }^{d}$ Calculated from experimental data (Ref. 40), assuming that $\Delta_{0}(H)=0.99$ $\mathrm{cm}^{-1}$ (Refs. 6, 38).

Reference 40.

(3) The $\mathrm{O} \cdots \mathrm{O}$ stretching fundamental is lower in $S_{1}$ than $S_{0}$, consistent with a smaller force constant in the excited state. ${ }^{44}$

(4) In agreement with experimental work, ${ }^{6}$ our data show little change in $\nu^{*}$ as $n$ increases, indicating the $\mathrm{O} \cdots \mathrm{O}$ stretching vibration can be treated approximately as harmonic in the $S_{1}$ state, even more so than in $S_{0}$. A similar result was obtained in experiments by Fuke $e t a l .^{45}$ for azaindole dimer.

(5) Our calculations suggest that excitation to the $S_{1}$ state should diminish $\mathrm{OH}$ and $\mathrm{OD}$ stretching and bending frequencies. This result agrees with experimental data, obtained for other $\mathrm{O}-\mathrm{H} \cdots \mathrm{O}$ systems, governing intramolecular excited state PT: $o$-hydroxyacetophenone ${ }^{46}$ and 1,8-dihydroxyanthraquinone. ${ }^{47}$

One can see from Table IV that the tunnel splittings are highly mode specific and the deformation vibrations do not couple strongly with PT motion in the $S_{1}$ state of tropolone, similar to the $S_{0}$ state. This arises from the fact that bending vibrations play a minor role in modulation of the PT barrier (see previous section). This result implies that correct description of the experimental regularities of PT in both electronic states of tropolone can be obtained in terms of the two-dimensional stretching model, which includes $\mathrm{O} \cdots \mathrm{O}$ and $\mathrm{O}-\mathrm{H}$ stretching vibration coordinates ${ }^{9-13}$ only.

Some of the trends noted here may be understood from Fig. 3 which illustrates schematically a number of proton transfer profiles at various values of $R(O O)$. Specifically, each double-well potential represents a section of $U_{0}^{*}(x, R)$ at constant $R$. These curves are layered over an overall blanket potential which represents the dependence of the system upon interoxygen distance in the excited electronic state. The energy levels $\epsilon_{0 v}^{*}$ are indicated in each double-well potential. $\epsilon_{01}^{*}$ and $\epsilon_{00}^{*}$ correspond to the energies of the two lowest proton vibrational states within any of the proton transfer potentials. These two quantities are illustrated by the curves in Fig. 4 as functions of $R$. For large interoxygen distances, these two energies are nearly identical, differing only as the two oxygens are brought closer together. This behavior can be attributed to the lower (and narrower) barrier for proton transfer as $R$ diminishes, more favorable for tunnel splitting, cf. Fig. 2.

It is the functional dependence of these two energy curves as $R$ varies that is traced out by the potentials in Fig. 4 . The energy levels of the $\mathrm{O} \cdots \mathrm{O}$ stretching vibrations, corresponding to these curves, are illustrated by solid $(v=0)$ curve or dashed $(v=1)$ horizontal lines in Fig. 4 . Were the curves in Fig. 4 parabolas with different force constants, the $\nu_{n}$ energy spacing between levels of like quantum number $v$ would remain constant as $n$ increases and $\Delta_{00 n}$ would increase monotonically and uniformly. The insensitivity of $\nu_{n}$ to $n$ in Tables III and IV suggests that these potentials are in fact well approximated by parabolas up to some small $n$. In the case where the potential is less harmonic and more Morse-like, the energy spacings $\nu_{n}$ diminish with $n$, so the splittings would likewise not grow as quickly as in the harmonic situation.

Despite its ability to reproduce some of the experimentally observed trends, the calculated PES for the $S_{1}$ state is less successful with other features of the excited state tropolone dynamics:

(a) The tunnel splitting of the vibrationless $\mathrm{O} \cdots \mathrm{O}$ stretch is calculated to be several times larger for the excited state than for $S_{0}$, whereas the experimental ratio is in excess of ten;

(b) The experimental tunnel splittings increase irregularly with $\mathrm{O} \cdots \mathrm{O}$ excitation in $S_{1}$, while a more regular trend is computed.

The calculated results imply that precise estimation of the PES of tropolone in the $S_{1}$ state can be obtained only with very extensive basis sets ( $f$ functions on $O$ and $d$ on bridged $\mathrm{H}$ ) with explicit account of correlation energy. The next section attempts to overcome these limitations via a model PES.

\section{Model PES for the $S_{1}$ excited state}

Previous papers devoted to theoretical study of gas phase PT in the multidimensional PES of systems with a symmetric AHA fragment ${ }^{3,4,10}$ have obtained the result that the tunnel splitting is increased monotonically and uniformly with vibrational excitation of the low-frequency mode. On the other hand, these theoretical models did not reproduce the fact that $\Delta_{00 n}^{*}$ passes through a maximum at small $n(n \leqslant 3)$, observed for TRN-OH. ${ }^{40}$ In order to clarify the role of various aspects of the PES, a model two-dimensional PES was constructed using analytical functions fit to the ab initio CIS data and our above conclusion that, to a good approximation, bending vibrations can be neglected in the tunnel splittings calculations.

The first goal was reproduction of the experimentally observed large value of the vibrationless level tunnel splitting in the $S_{1}$ state, in comparison with that in the $S_{0}$ state for TRN-OH, by adjusting the barrier height [via parameter $\lambda_{1}$ in Eq. (A5)]. A good value of $\Delta_{0}^{*}$ could be extracted when $\lambda_{1}$ was set to $118 \mathrm{kcal} / \mathrm{mol}$ which leads to a "frozen" PT barrier of $14.3 \mathrm{kcal} / \mathrm{mol}$ at $R_{e}^{*}$ [Fig. 3(a) corresponds approximately to such a barrier height but with $\left.R_{e}^{*}=2.52 \AA\right]$, compared to the $a b$ initio CIS value of $24 \mathrm{kcal} / \mathrm{mol}$. The experimental 
TABLE V. Calculated values of the relative energies and tunnel splittings for the model 2-D PES representing the $S_{1}$ state of TRNOH and TRNOD. Available experimental data are given in parentheses. All values in $\mathrm{cm}^{-1}$.

\begin{tabular}{|c|c|c|c|c|c|}
\hline \multicolumn{2}{|c|}{ Quantum Nos. } & \multicolumn{2}{|c|}{ Relative energy, $E_{v n}$} & \multicolumn{2}{|c|}{ Tunnel splitting, $\Delta_{v n}{ }^{a}$} \\
\hline$v$ & $n$ & $\mathrm{H}$ & D & $\mathrm{H}$ & $\mathrm{D}$ \\
\hline 0 & 0 & 0 & 0 & $22.3\left(19.92^{d}\right)$ & $2.6\left(2.0^{\mathrm{e}}\right)$ \\
\hline .0 & 1 & $338\left(302^{b}\right)$ & $344\left(299^{c}\right)$ & $44.7\left(30.99^{d}\right)$ & $7.6\left(11.0^{2}\right)$ \\
\hline 0 & 2 & 664 & 660 & $50.1\left(28.99^{d}\right)$ & $10.8\left(13.0^{e}\right)$ \\
\hline 0 & 3 & 925 & 900 & 39.7 & 10.0 \\
\hline
\end{tabular}

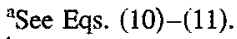

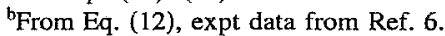

From Eq. (12), expt data from Ref. 40.

${ }^{d}$ Calculated from experimental data (Ref. 40), assuming that $\Delta_{0}(H)=0.99$ $\mathrm{cm}^{-1}$ (Refs. 6, 38).

${ }^{\mathrm{e}}$ Reference 40.

value of the $\mathrm{O} \cdots \mathrm{O}$ stretch fundamental frequency of TRN-OH is reproduced fairly well with the model $W^{*}$ potential [see Eqs. (A7) and (15)], which is characterized by a much lower $k_{\sigma}$ in comparison with the CIS calculations.

Energy levels $E_{v n}$ calculated using the 2-D model PES are reported in Table $\mathrm{V}$, along with the splittings. The model PES reproduces the main features of the excited state tropolone dynamics:

(i) the tunnel splittings are in much better agreement with experiment compared to the $a b$ initio CIS values; the $\Delta_{0}^{*}$ quantity for $\mathrm{H}$ is only one order of magnitude larger than that for $D$ in Table $V$ whereas the ratio is much larger for the CIS potential in Table IV. This discrepancy can be attributed to the lower and narrower proton transfer barrier in the case of the model potential, which would tend to diminish the frequency deuterium isotope effect, as in Fig. 4 of Ref. 11. Accordingly, the experimental tunnel splitting of the excited $\mathrm{O} \cdots \mathrm{O}$ stretch levels differ in magnitude of 2 to 3 times that for $\mathrm{H}$ and $\mathrm{D}$ species (Table $\mathrm{V}$ ) and the calculated values are in agreement with this;

(ii) $\Delta_{0 n}^{*}$ for $\mathrm{TRN}-\mathrm{OH}$ passes through a maximum at small $n(n \leqslant 3)$. A similar result was obtained in Ref. 13 where the calculated tunneling transition probabilities (for the $S_{0}$ state) appeared to pass through a maximum at $n=2$. At the same time the calculated tunnel splitting for different $n$ in Refs. 3, 4, and 10 has no maximum;

(iii) The $\mathrm{O} \cdots \mathrm{O}$ stretch vibrations for TRNOH can be approximately treated as harmonic for $n \leqslant 3$.

According to Eq. (A7), the model potential $\epsilon_{v}^{*}(v=0,1)$ is presented as a sum of the $\mathrm{O} \cdots \mathrm{O}$ interaction energy function $W^{*}(R)$ and proton adiabatic energy $\mathrm{e}_{v}^{*}(R)$. The former can be supposed to be a harmonic function for small deviation from equilibrium (for small $n$ )

$$
W^{*}(R) \approx\left(\frac{1}{2}\right) k_{\sigma}\left(R-R_{0}\right)^{2} .
$$

This function, together with $\mathrm{e}_{0}^{*}(R)$ and $\mathrm{e}_{i}^{*}(R)$ are presented in Fig. 5. As seen in this figure, $\mathrm{e}_{0}^{*}(R)$ and $\mathrm{e}_{1}^{*}(R)$ intersect at $R^{* *} \approx 2.55 \AA$. At $R>R^{* * *}$, the united curve $\mathbf{e}_{0,1}^{*}(R)$ depends

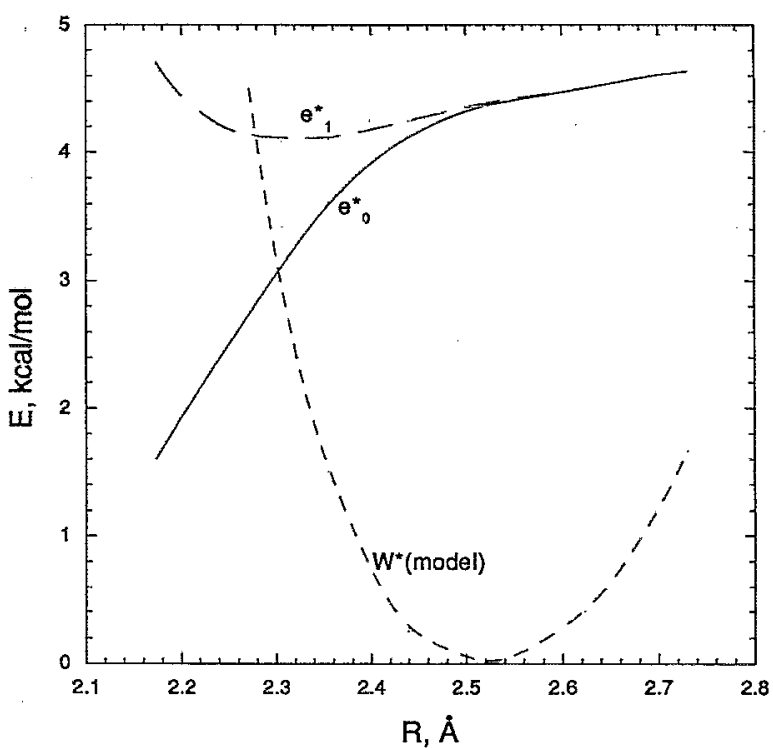

FIG. 5. Energies of the two lowest proton stretch levels $\mathrm{e}_{v}^{*}$ and the $W^{*}$ potential as functions of $R$.

quadratically on $R$ with negative curvature. ${ }^{13}$ According to Eq. (11) of Ref. 13, this dependence for the $\mathrm{OH} \cdots \mathrm{O}$ fragment is

$$
\mathrm{e}_{0,1}^{*}(R)=\left(\frac{1}{2}\right) h \nu(\mathrm{OH})-\left(\frac{1}{2}\right) a_{0}(R-2.90)^{2},
$$

where $R$ is in $\AA, a_{0}=8.3 \times 10^{3} \mathrm{~cm}^{-1} \AA^{2}$ (there is a misprint in Ref. 13). This function may be extrapolated a little in the $R<R^{* *}$ region. For this reason the $\epsilon_{0}^{*}(R)$ function retains its harmonic character with apparent force constant $k_{\sigma}^{0}(\mathrm{app})=k_{\sigma}-a_{0}\left(k_{\sigma}>a_{0}\right)$.

In contrast to $\mathbf{e}_{0}^{*}$, the $\mathbf{e}_{1}^{*}$ curve has a point of inflection at $R \leqslant R^{* *}$ (Fig. 5) and for this reason its curvature is very small in the vicinity of the equilibrium point. This means the apparent force constant is $k_{\sigma}^{1}($ app $) \approx k_{\sigma}>k_{\sigma}^{0}($ app $)$. Hence, the $\mathbf{e}_{0}^{*}(R)$ and $\mathbf{e}_{1}^{*}(R)$ functions can be represented by two almost parabolic curves similar to that presented in Fig. 4. One can see that the frequencies $\nu_{n}$ are almost constant while the tunnel splitting $\Delta_{00 n}$ increases with $n$ at sufficiently small $n$. This regularity is changed when anharmonicity begins to play a role. As our $a b$ initio calculations show, similar consideration is possible for the ground state.

\section{DISCUSSION}

Theoretical two-dimensional PESs for the $S_{0}$ and $S_{1}$ electronic states of heteroaromatic molecules governing excited-states proton transfer have not been published yet, except for salicylic acid ${ }^{48}$ and 3-hydroxyflavone. ${ }^{49}$ However, the latter surfaces were obtained using the very approximate CNDO/2 and MNDO/AM1 (with limited CI) methods, respectively. We will focus instead on the $a b$ initio calculations and on the model two-dimensional PESs, which have been developed for the ground state of systems with a symmetric $\mathrm{OH} \cdots \mathrm{O}$ fragment.

Redington ${ }^{35}$ developed his model two-dimensional PES for PT in the ground state of tropolone on the basis of avail- 
able infrared frequencies, tunnel splittings, and H/D isotope effects. He adopted the philosophy that it is principally tunneling of the heavy atoms that are at the root of experimental observations and consequently took these heavy atom motions as the prime variables, while the motions of the $\mathrm{H}$ atom were relegated to the set of 37 normal harmonic vibrations, subject to adiabatic separation, so as to obtain his reaction surface. The relaxed proton transfer barrier was found to be equal to $16.0 \mathrm{kcal} / \mathrm{mol}$, similar to the value obtained here $(15.7 \mathrm{kcal} / \mathrm{mol})$. Redington found that the tunnel splittings are highly mode specific, in agreement with our own conclusions. On the other hand, the absence of $\mathrm{H}$ modes in the reaction surface is troubling if one hopes to understand the PT dynamics. For instance, this approximation leads to the incorrect conclusion that vibrationless level tunneling splittings are similar for $\mathrm{H}$ and $\mathrm{D}$ tropolone.

Another approach was explored by Bosch et al. ${ }^{50}$ in their work with malonaldehyde and the hydrogenoxalate anion. The potential surface was considered as the sum of a doublewell potential function of $x$ (dominant component of which is the displacement of transferring $\mathrm{H}$ ), and a harmonic function of another variable $y$ (the dominant component of which is the $O \cdots O$ stretching vibrations), orthogonal but strongly coupled to $x$. The authors assumed quadratic coupling between $y$ and $x$ and included four adjustable parameters in their potential as a fit to the $a b$ initio PES. The calculated values of the vibrationless level tunnel splitting $\left(1.3 \mathrm{~cm}^{-1}\right)$ and fundamental frequency of the $0 \cdots O$ stretch $\left(364 \mathrm{~cm}^{-1}\right)$ for hydrogenoxalate anion, see Table VIII in Ref. 50, are very close to the experimental result for its close analogtropolone. The energy levels corresponding to the excited states of $x$ motion (OH stretch) are not given in Ref. 50. The H/D isotope effect on the tunnel splitting was not studied in Ref. 50. In the later paper ${ }^{51}$ the same authors calculated the $\mathrm{H} / \mathrm{D}$ effect, but in terms of a one-dimensional model.

A prime attractive feature of the localized $\mathrm{OHO}$ fragment model is its relative simplicity and provision of a clear picture in terms of modes. For example, levels can be assigned easily even in the case of high density of vibrational levels. (Such assignment may be easy if the Schrödinger equation is solved directly with multidimensional potentials, e.g., Fig. 4 in Ref. 10). In contrast, excited vibrational states of the reaction surface subspace do not really correspond to states of the calculated system, ${ }^{3,4}$ due to the difference in choice of the coordinate systems used in Wilson's GF matrix method and the reaction surface model.

The two-dimensional stretching model, suggested in the present paper for the description of PT in the ground and first excited singlet state of tropolone, agrees with the results of the recent femtosecond studies of excited state PT in 2-(2-hydroxyphenyl)benzothiazole ${ }^{52}$ and methylsalicylate. ${ }^{53}$ According to Refs. 52 and 53 the PT in these compounds is the result of coupling between $\mathrm{O}-\mathrm{H}$ stretch and some lowfrequency coordinate.

\section{CONCLUSIONS}

The model of the localized $\mathrm{OH} \cdots \mathrm{O}$ fragment, combined with $a b$ initio SCF calculations, provides a quantitatively correct picture of hydrogen bonding and proton transfer in the $S_{0}$ state of tropolone. The $a b$ initio CIS calculations reproduce the principal experimental trends established for $S_{1} \leftarrow S_{0}$ excitation in tropolone, but are less successful with some other features of the dynamics of excited state tropolone. Tunnel splittings of the proton stretch levels are found to be highly mode specific. In contrast to the $0 \cdots O$ stretching vibrations, excitation of the $\mathrm{OH}$ bending vibra-. tions have much weaker effects on the tunnel splittings. Thus, a sufficiently correct description of the tunnel splittings of the proton stretch levels in the two lowest singlet electronic states of tropolone can be obtained in terms of the two-dimensional stretching model, which includes $\mathrm{O} \cdots \mathrm{O}$ and $\mathrm{O}-\mathrm{H}$ stretching vibration coordinates only. Proper behavior of the excited electronic state PT in gas-phase tropolone seems to involve a relatively low potential barrier (only one doublet of the $\mathrm{OH}$ stretch lies under the peak) and the PT occurs in the vicinity of the top, requiring probably excitation of not more than one or two $\mathrm{O} \cdots \mathrm{O}$ stretch levels.

\section{ACKNOWLEDGMENTS}

This work was supported by the U.S. National Science Foundation (CHE-9123824) and by the CAST Program of the National Research Council.

\section{APPENDIX A}

Following Refs. 10, 11, and 13, we assume that the $U_{m}(x, R)$ function of Eq. (6), depending on $R$ parametrically, can be written as

$$
U_{m}(x, R)=V_{m}(x, R)+W(R),
$$

where $V_{m}(x, R)$ is an adiabatic potential for the "fast" subsystem and $W(R)$ is the $\mathrm{O} \cdots \mathrm{O}$ interaction energy. $V_{m}(x, R)$ is approximated by the function, designated as $V$, and suggested in Ref. 11. It has some essential advantages over other model functions which are usually used in numerical calculations of $\mathrm{PT}$ in systems with the symmetric $\mathrm{OH} \cdots \mathrm{O}$ fragment, see, e.g. Ref. 54. In particular, $V$ is more flexible than the combination of two Morse functions ${ }^{10}$ or polynomials, ${ }^{50}$ as it enables the barrier to be varied independently from the lateral parts of the potential; $V$ is characterized by only three fitting parameters (at a given $R$ value), in contrast to a Lippincott-Schroeder function, ${ }^{55}$ each with a clear physical meaning. It was shown in the Results Section, that the bending vibration plays a minor role in modulation of the frozen barrier of PT in both electronic states; it can therefore be neglected in the calculations of the proton stretch level tunnel splittings.

Following Refs. 11 and 13 , we assume that $V(x, R)$ consists of three parts. The central part represents the potential barrier of width $2 b$ and height $V_{0}$, and the two surrounding parts describe the proton repulsion from the left and right oxygen atoms:

$$
\begin{aligned}
& V(x, R)=V_{0}(b) \cos ^{2}(\pi x / 2 b), \quad|x| \leqslant b, \\
& V(x, R)=\frac{1}{2} k(b)(|x|-b)^{2}, \quad|x| \geqslant b .
\end{aligned}
$$

The right side of these equations do not contain $R$ explicitly but they depend on $R$ through parameter $b$. We replace $R$ 
TABLE VI. Characteristic values of the parameters for $V$.

\begin{tabular}{ccccccc}
\hline \hline & $\begin{array}{c}b_{0} \\
\AA\end{array}$ & $b_{1}$ & $\begin{array}{c}\lambda_{0} \\
\mathrm{kcal} / \mathrm{mol}\end{array}$ & $\begin{array}{c}\lambda_{1} \\
\mathrm{kcal} / \mathrm{mol} \AA^{-2}\end{array}$ & $\begin{array}{c}k_{0} \\
\mathrm{kcal} / \mathrm{mol} \AA^{-2}\end{array}$ & $\begin{array}{c}k_{1} \\
\mathrm{kcal} / \mathrm{mol} \AA^{-3}\end{array}$ \\
\hline$S_{0}$. & -0.998 & 0.552 & -2.49 & 205.4 & 1210.0 & 56.2 \\
$S_{1}$ & -1.029 & 0.561 & -3.25 & 182.7 & 1140.0 & 56.6 \\
\hline \hline
\end{tabular}

with $b$ in order to obtain Eqs. (A2) in compact form similar to those used in Refs. 11 and 13. The value of $b$ can be determined approximately as

$$
b=r^{\prime}-r_{e}
$$

where $r^{\prime}$ and $r_{e}$ denote the $\mathrm{OH}$ distance for the $C_{2 v}$ and $C_{s}$ structures, respectively, at a given $R$ (see Fig. 1). To a first approximation, $b$ can be treated as a function of $R$ only. It was learned that $b$ depends linearly on $R$

$$
b=b_{0}+b_{1} R \text {. }
$$

$V_{0}$ was found to depend on $b$ quadratically in both electronic states

$$
V_{0}=\lambda_{0}+\lambda_{1} b^{2}
$$

and $k$ varies linearly with $R \leqslant R_{e}$ and can be treated as approximately constant for $R>R_{e}$ :

$$
\begin{aligned}
& k=k_{0}+k_{1} b, \quad R \leqslant R_{e}, \\
& k=\text { const }, \quad R>R_{e} .
\end{aligned}
$$

The values of the parameters $a_{0}, a_{1}, k_{0}, k_{1}, b_{0}$, and $b_{1}$, for the $S_{0}$ and $S_{1}$ states were determined from our quantum mechanical data and are listed in Table VI.

After solving Eq. (6) with the model potential energy function $V$ we obtain proton (deuteron) adiabatic levels $\mathbf{e}_{0}(R)$. Using Eq. (A1), the model potential for $\mathrm{O} \cdots \mathrm{O}$ stretching vibration $\epsilon_{v}(R)$ can be written as

$$
\epsilon_{v}(R)=\mathbf{e}_{v}(R)+W(R),
$$

where the index $m$ is omitted, because of the neglect of the bending vibrations in the model PES.

\section{APPENDIX B}

As verification of Eqs. (6) and (7), consider the linear triatomic system AH $\cdots$ A. Following Ref. 10, its Hamiltonian can be written as

$$
\begin{gathered}
{\left[-\frac{\hbar^{2}}{2}\left\{\left(\frac{1}{m}+\frac{1}{M}\right) \frac{\partial^{2}}{\partial x^{2}}+\left(\frac{1}{M}+\frac{1}{M}\right) \frac{\partial^{2}}{\partial R^{2}}+\frac{2}{M} \frac{\partial^{2}}{\partial x \partial R}\right\}\right.} \\
+V(x, R)+W(R)] \Psi(x, R)=E \Psi(x, R),
\end{gathered}
$$

where $R$ is the A $\cdots \mathrm{A}$ interatomic distance and $x$ is the distance from $\mathrm{H}$ to the midpoint between the A atoms; $m$ and $M$ are the masses of $\mathrm{H}$ and $\mathrm{A}$, respectively. The functions $V(x, R)$ and $W(R)$ have the same meaning as in Eq. (A1), and $W(R)$ is assumed to be harmonic.

We calculated the level spectrum of the simplified Hamiltonian derived from Eq. (B1) by omitting the off-
TABLE VII. A $\cdots$ A stretch frequencies and corresponding tunnel splittings obtained using Stepanov approximation and simplified Hamiltonians with the model 2-D PES of the AHA fragment (Ref. 10). Exact values from Fig. 4 in Ref. 10 are given in parentheses. All values in $\mathrm{cm}^{-1}$.

\begin{tabular}{cccc}
\hline \hline Quantum Nos. & Relative energy, $E_{v n}$ & Tunnel splitting, $\Delta_{v n}{ }^{\mathrm{n}}$ \\
\hline 0 & $n$ & & \\
0 & 0 & 0 & $98(111)$ \\
0 & 1 & $408^{\mathrm{b}}(400)$ & $116(133)$ \\
0 & 2 & $409(403)$ & $134(151)$ \\
0 & 3 & $409(408)$ & $150(166)$ \\
0 & 4 & $411(398)$ & $160(193)$ \\
0 & 5 & $412(416)$ & $180(202)$ \\
\hline
\end{tabular}

${ }^{\mathrm{a}}$ See Eq. (10).

${ }^{b}$ From Eq. (12).

diagonal element in the kinetic part. Using adiabatic separation of the $x$ and $R$ variables, one can write the wave equation for $H$ motion as

$$
H_{1} \phi_{v}(x, R)=\mathbf{e}_{v}(R) \phi_{v}(x, R),
$$

where the simplified Hamiltonian is

$$
H_{1}=-\frac{\hbar^{2}}{2}\left(\frac{1}{m}+\frac{1}{M}\right) \frac{\partial^{2}}{\partial x^{2}}+V(x, R) .
$$

The wave equation for $\mathrm{A} \cdots \mathrm{A}$ stretching vibrations is

$$
H_{2} \chi_{v n}(R)=E_{v n} \chi_{v n}(R),
$$

where the simplified Hamiltonian is

$$
H_{2}=-\frac{\hbar^{2}}{2}\left(\frac{1}{M}+\frac{1}{M}\right) \frac{\partial^{2}}{\partial R^{2}}+\epsilon_{v}(R) .
$$

The effective A $\cdots$ A stretching potential $\epsilon_{\eta}$. associated with the $\nu$ th proton stretch level can be written as

$$
\epsilon_{v}(R)=\mathbf{e}_{v}(R)+W(R) .
$$

The calculated energy levels and corresponding splittings for $v=0$ and 1 are given in Table VII. "Approximate" A $\cdots$ A stretch frequencies agree with the exact ones very well, while the approximate values of the tunnel splittings are a little lower than the exact values; the average relative difference is ca. $12 \%$.

${ }^{1}$ T. Tsuji, H. Sekiya, Y. Nishimura, R. Mori, A. Mori, and H. Takeshita, J. Chem. Phys. 97, 6032 (1992), and references therein.

${ }^{2}$ T. Carrington and W. H. Miller, J. Chem. Phys. 84, 4364 (1986).

${ }^{3}$ N. Shida, P. F. Barbara, and J. Almlöf, J. Chem. Phys. 91, 4061 (1989).

${ }^{4}$ N. Shida, P. F. Barbara, and J. Almlöf, J. Chem. Phys. 94, 3663 (1991).

${ }^{5}$ B. I. Stepanov, Zh. Fiz. Khim. 19, 507 (1945); 20, 907 (1946). The Stepanov approximation is similar to the Born-Oppenheimer separation of the electronic and nuclear motions: M. Born and J. Oppenheimer, Ann. Phys. 84, 457 (1927); M. Born, K. Huang, in Dynamical Theory of Crystal Lattices, Appendix 8 (Oxford University, Oxford, 1954).

${ }^{6} \mathrm{H}$. Sekiya, Y. Nagashima, T. Tsuji, Y. Nishimura, A. Mori, and $\mathrm{H}$. Takeshita, J. Phys. Chem. 95, 10311 (1991).

${ }^{7}$ R. Meyer and R. R. Ernst, J. Chem. Phys. 93, 5518 (1990).

${ }^{8}$ N. Shida, J. Almlof, and P. F. Barbara, J. Phys. Chem. 95, 10457 (1991).

${ }^{9}$ N. D. Sokolov and V. A. Savel'ev, Chem. Phys. 22, 383 (1977), and references therein.

${ }^{10}$ N. Sato and S. Iwata, J. Chem. Phys. 89, 2932 (1988).

${ }^{11}$ N. D. Sokolov, M. V. Vener, and V. A. Savel'ev, J. Mol. Struct. 222, 365 (1990).

${ }^{12}$ M. V. Vener, Chem. Phys. 166, 311 (1992).

${ }^{13}$ N. D. Sokolov and M. V. Vener, Chem. Phys. 168, 29 (1992). 
${ }^{14} \mathrm{H}$. Azzouz and D. Borgis, J. Chem. Phys. 98, 7361 (1993), and references therein.

${ }^{15}$ N. D. Sokolov and V. A. Savel'ev. Chem. Phys. 181, 305 (1994).

${ }^{16}$ Using polar coordinates for representation of vector $\mathbf{r}$ we define Jacobi relative coordinates $\left(\mathbf{r}, \mathbf{R}^{J a c}, \theta^{J a c}\right)$ for $\mathrm{O}-\mathrm{H} \cdots \mathrm{O}$ fragment as follows: $\mathbf{r}$ connects $\mathrm{A}$ and $\mathrm{H}$ atoms, $\mathbf{R}^{\text {Jac }}$ gives the position of the $\mathrm{B}$ atom with respect to the center of mass of the $\mathrm{AH}$ pair and $\theta^{J a c}$ is the angle between $\mathbf{r}$ and $\mathbf{R}^{J a c} \cdot \mathbf{R}^{J a c}$ can be replaced by $\mathbf{R}$ (the $\mathrm{A} \cdots \mathrm{B}$ interatomic vector) and $\theta^{J a c}$ by $\theta$, where $\theta$ is the angle between $\mathbf{r}$ and $\mathbf{R}$. For the equilibrium tropolone geometry in the $S_{0}$ state one finds $r=0.953 \AA, R^{J a c}=2.488 \AA$, $\theta^{J a c}=43.4^{\circ} ; R=2.529 \AA, \theta=42.6^{\circ}$.

${ }^{17}$ S. A. Barton and W. R. Thorson, J. Chem. Phys. 71, 4263 (1979).

${ }^{18}$ T. R. Singh and J. L. Wood, J. Chem. Phys. 48, 4567 (1968).

${ }^{19}$ Qian Thi-Ding, Zhang Xing-Guo, Li Xing-Wen, H. Kono, and S. H. Lin, Mol. Phys. 47, 713 (1982).

${ }^{20}$ J. Manz, R. Meyer, and J. Romelt, Chem. Phys. Lett. 96, 607 (1983).

${ }^{21}$ G. S. Ezra, Chem. Phys. Lett. 101, 259 (1983).

${ }^{22}$ B. R. Johnson, R. T. Skodje, and W. P. Reinhard, Chem. Phys. Lett. 112, 396 (1984).

${ }^{23}$ J. Manz, R. Meyer, E. Pollak, J. Romelt, and H. H. R. Schor, Chem. Phys. 83, 333 (1984).

${ }^{24}$ M. R. Sage, Chem. Phys. Lett. 93, 453 (1985).

${ }^{25}$ C. Kubash, G. Nguyen Vien, and M. Richard-Viard, J. Chem. Phys. 94, 1929 (1991).

${ }^{26}$ B. Grace and R. T. Skodje, J. Chem. Phys. 95, 7249 (1991).

${ }^{27}$ J. B. Foresman, M. Head-Gordon, J. A. Pople, and M. J. Frish, J. Phys. Chem. 96, 135 (1992).

${ }^{28}$ GAUSSIAN 92, Revision A, M. J. Frish, G. W. Trucks, M. Head-Gordon, P. M. W. Gill, M. W. Wong, J. B. Foresman, B. G. Johnson, H. B. Shclegel, M. A. Robb, E. S. Replogle, R. Comperts, J, L. Andres, K. Raghavachari, J. S. Binkley, C. Gonzalez, R. L. Martin, D. J. Fox, D. J. Defrees, J. Baker, J. J. P. Stewart, and J. A. Pople (Gaussian, Inc., Pittsburgh, 1992).

${ }^{29}$ W. J. Hehre, L. Radom, P. v. R. Schleyer, and J. A. Pople, Ab initio Molecular Orbital Theory (Wiley, New York, 1986).

${ }^{30}$ N. D. Sokolov, M. V. Vener, and V. A. Savel'ev, J. Mol. Struct. 177, 93 (1988).

${ }^{31}$ B. R. Johnson, J. Chem. Phys. 67, 4086 (1977).
${ }^{32}$ R. L. Redington and C. W. Bock, J. Phys. Chem. 95, 10284 (1991).

${ }^{33}$ J. Shimanouchi and Y. Sasada, Acta Crystallogr. B 81, 29 (1973).

${ }^{34}$ A. C. P. Alves and J. M. Hollas, Mol. Phys. 23, 927 (1972).

${ }^{35}$ R. L. Redington, J. Chem. Phys. 92, 6447 (1990).

${ }^{36}$ N. Sanna, F. Ramondo, and L. Bencivenni, J. Mol. Struct. 318, 217 (1994).

${ }^{37}$ S. L. Baughcum, R. W. Duerst, W. F. Rowe, Z. Smith, and E. B. Wilson, J. Am. Chem. Soc. 103, 6296 (1981).

${ }^{38}$ H. Honjo, H. Onaka, K. Tanaka, and T. Tanaka, Proceedings of the 12 th Colloquium on High-Resolution Molecular Spectroscopy (Dijon, France, 1991).

${ }^{39}$ A. C. P. Alves, J. M. Hollas, H. Musa, and T. Ridley, J. Mol. Spectrosc. 109, 99 (1985).

${ }^{40}$ H. Sekiya, Y. Nagashima, and Y. Nishimura, J. Chem. Phys. 92, 5761 (1990).

${ }^{41}$ R. L. Redington and T. E. Redington, J. Mol. Spectrosc. 78, 229 (1979).

${ }^{42}$ Y. Tomioka, M. Ito, and N. Mikami, J. Phys. Chem. 87, 4401 (1983).

${ }^{43}$ R. L. Redington, Y. Chen, G. J. Scherer, and R. W. Field, J. Chem. Phys. 88, 627 (1988).

${ }^{44}$ R. L. Redington, T. E. Redington, M. A. Hunter, and R. W. Field, J. Chem. Phys. 92, 6456 (1990).

${ }^{45} \mathrm{~K}$. Fuke and K. Kaya, J. Phys. Chem. 93, 614 (1989).

${ }^{46}$ T. Nishiya, S. Yamauchi, N. Hirota, M. Baba, and I. Hanazaki, J. Phys. Chem. 90, 5730 (1986).

${ }^{47}$ M. H. Van Benthem and G. D. Gillispie, J. Phys. Chem. 88, 2954 (1984).

${ }^{48} \mathrm{~J}$. Catalan and J. I. Fernandez-Alonso, J. Mol. Struct. 27, 59 (1975).

${ }^{49}$ A. Muhlpfordt, T. Bultmann, N. P. Ernsting, and B. Dick, Chem. Phys. 181, 447 (1994).

${ }^{50}$ E. Bosch, M. Moreno, J. M. Lluch, and J. Bertran, J. Chem. Phys. 93, $5685(1990)$.

${ }^{51}$ E. Bosch, M, Moreno, and J. M. Lluch, Can. J. Chem. 70, 100 (1992)

${ }^{52} \mathrm{~F}$. Laermer, T. Elsaesser, and W. Kaiser, Chem. Phys. Lett. 148, 119 (1988).

${ }^{53}$ J. L. Herek, S. Pedersen, L. Banares, and A. H. Zewail, J. Chem. Phys. 97, 9046 (1992).

${ }^{54}$ X. Duan and S. Scheiner, J. Mol. Struct. 270, 173 (1992).

${ }^{55}$ T. Saitoh, K. Mori, and R. Itoh, Chem. Phys. 60, 161 (1981). 